\title{
Active Power Measurement Based on Multiwavelet Transforms
}

\author{
Xiao-Bing Zhang, Yun-Hui Li, and Xiao-Meng Cui \\ School of Measurement and Communication, Harbin University of Science and Technology, Harbin 150080, China \\ Correspondence should be addressed to Xiao-Bing Zhang; zhangxb_hust@163.com
}

Received 29 June 2013; Revised 31 December 2013; Accepted 2 January 2014; Published 11 February 2014

Academic Editor: Claudio R. Fuerte-Esquivel

Copyright (C) 2014 Xiao-Bing Zhang et al. This is an open access article distributed under the Creative Commons Attribution License, which permits unrestricted use, distribution, and reproduction in any medium, provided the original work is properly cited.

\begin{abstract}
This paper discusses a new method for calculating active power in the multiwavelet domain. When the voltage and current waveforms are analyzed using multiwavelet, the active power can be calculated by simply adding the products of the multiwavelet coefficients without having to reconstruct the signals back to the time domain first and then using the traditional integration. From the simulation result, we can see that the results using multiwavelet are better than the ones using wavelet and Fourier Transforms no matter which prefilter is used.
\end{abstract}

\section{Introduction}

Active power is important for many purposes such as designing power system equipment, setting tariffs, developing measurement meters, and designing compensation devices for improving the quality of the electric energy. Under sinusoidal conditions, the definitions of active power work well. However, due to the recent widespread use of nonlinear loads, the voltage and current waveforms become nonsinusoidal and therefore their traditional definitions become unsuitable. As a result, many attempts have been made to define active power under this new situation [1-5].

Wavelet is an effective tool for nonstational signal processing and has been used in the measurement of active power [6-8]. However, scalar wavelets cannot contain orthogonality, symmetry, compact support, and higher order of vanishing moments simultaneously. Multiwavelet transform is a new concept in the framework of wavelet transform but has some important differences. It simultaneously possesses orthogonality, compact support, higher order of vanishing moments, and symmetry. It has become a tool for power quality study recently [9-11].

In this paper, a new approach to measure active power based on multiwavelet transforms is studied. In Section 2, we introduce the multiwavelet transform. In Section 3, a new approach to measure active power based on multiwavelet transforms is discussed. In Section 4, an example is given to illustrate validity of our method. At last a conclusion is given in Section 5.

\section{Multiwavelet Transform}

Unlike a scalar wavelet, a multiwavelet has several mother wavelet functions (scaling functions) which are used to expand a given function [12]. Let $\Phi$ denote a compactly supported orthonormal scaling vector $\Phi=\left(\phi_{1}, \ldots, \phi_{r}\right)^{T}$, where $r$ is the number of scalar scaling functions. Then $\Phi(t)$ satisfies a two-scaling dilation equation of the form

$$
\Phi(t)=\sum_{k} H_{k} \Phi(2 t-k)
$$

for some finite sequence $H$ of $r \times r$ matrices. Furthermore, the integer shifts of the components of $\Phi$ form an orthonormal system; that is,

$$
\left\langle\phi_{n}(\cdot-k), \phi_{n^{\prime}}\left(\cdot-k^{\prime}\right)\right\rangle=\delta_{n-n^{\prime}, k-k^{\prime}} .
$$

Let $V_{0}$ denote the closed span of $\left\{\phi_{n}(\cdot-k) \mid k \in \mathbf{Z}, n=\right.$ $1,2, \ldots, r\}$ and define $V_{j}=\left\{f\left(2^{j} \cdot\right) \mid f \in V_{0}\right\}$. Then $\left(V_{j}\right)_{j \in \mathbf{Z}}$ is a multiresolution analysis of $L^{2}(\mathbf{R})$ [13]. Note that we choose the increasing convention $V_{j-1} \subset V_{j}$.

Let $W_{j}$ denote the orthogonal complement of $V_{j}$ in $V_{j+1}$. Then there exists an orthogonal multiwavelet $\Psi=$ $\left(\psi_{1}, \ldots, \psi_{r}\right)^{T}$ such that $\left\{\psi_{n}(\cdot-k) \mid n=1,2, \ldots, r\right.$ and $\left.k \in \mathbf{Z}\right\}$ forms an orthogonal basis of $W_{0}$. Since $W_{0} \subset V_{1}$, there exists a sequence $G$ of $r \times r$ matrices such that

$$
\Psi(t)=\sum_{k} G_{k} \Phi(2 t-k) .
$$


Let $f \in V_{0}$; then $f$ can be written as a linear combination of the basis in $V_{0}$. Consider

$$
f(t)=\sum_{k \in \mathbf{Z}} \mathbf{c}_{0}^{*}(k) \Phi(t-k)
$$

for some sequence $\mathbf{c}_{0} \in l_{2}(\mathbf{Z})^{r}$ of vector coefficients. The superscript $*$ stands for the complex conjugate transposition. Here $l_{2}(\mathbf{Z})^{r}$ denotes the space of finite energy vector sequences $\mathbf{c}$ with the norm

$$
\|\mathbf{c}\|=\left(\sum_{\substack{i=1, \ldots, r \\ n \in \mathbb{Z}}}\left|\mathbf{c}_{i}(n)\right|^{2}\right)^{1 / 2} .
$$

Because $V_{0}=V_{-1} \oplus W_{-1}$ [14], where the symbol $\oplus$ denotes an orthogonal direct sum, $f$ can be written as a linear combination of the basis functions of $V_{-1}$ and $W_{-1}$

$$
f(t)=\sum_{k \in \mathbf{Z}} \sqrt{2} \mathbf{c}_{1}^{*}(k) \Phi(2 t-k)+\sum_{k \in \mathbf{Z}} \sqrt{2} \mathbf{d}_{1}^{*}(k) \Psi(2 t-k) .
$$

The coefficients $\mathbf{c}_{1}$ and $\mathbf{d}_{1}$ are related to $\mathbf{c}_{0}$ via the following decomposition and reconstruction algorithm:

$$
\begin{gathered}
\mathbf{c}_{1}(k)=\sum_{n} H(n) \mathbf{c}_{0}(2 k+n), \\
\mathbf{d}_{1}(k)=\sum_{n} G(n) \mathbf{c}_{0}(2 k+n), \\
\mathbf{c}_{0}(k)=\sum_{n} H^{*}(k-2 n) \mathbf{c}_{1}(n)+\sum_{n} G^{*}(k-2 n) \mathbf{d}_{1}(n) .
\end{gathered}
$$

Define $\Phi_{l, k}(t)=2^{l / 2} \Phi\left(2^{l} t-k\right)$ and $\Psi_{l, k}(t)=2^{l / 2} \Psi\left(2^{l} t-k\right) ;$ then $\left\{\Phi_{l, k}: k \in \mathbf{Z}\right\}$ and $\left\{\Psi_{l, k}: k \in \mathbf{Z}\right\}$ are stable bases of $V_{l}$ and $W_{l}$, respectively. And (6) can be written as

$$
f(t)=\sum_{k \in \mathbf{Z}} \mathbf{c}_{1}^{*}(k) \Phi_{1, k}(t)+\sum_{k \in \mathbf{Z}} \mathbf{d}_{1}^{*}(k) \Psi_{1, k}(t) .
$$

Because $V_{0}=V_{-1} \oplus W_{-1}=\left(V_{-2} \oplus W_{-2}\right) \oplus W_{-1}=\cdots=$ $V_{-m} \oplus W_{-m} \oplus \cdots \oplus W_{-1}$, where $m$ is the levels for decomposition, $f$ can be written as

$$
f(t)=\sum_{k \in \mathbf{Z}} \mathbf{c}_{m}^{*}(k) \Phi_{m, k}(t)+\sum_{l=-m}^{-1} \sum_{k \in \mathbf{Z}} \mathbf{d}_{l}^{*}(k) \Psi_{l, k}(t) .
$$

In general, for a signal $f(t) \in V_{n}, n \in \mathbf{Z}$, it can be expressed as a linear combination of the basis in $V_{n}$. Consider

$$
f(t)=\sum_{k} \mathbf{c}_{n}^{*}(k) \Phi_{n, k}(t) .
$$

Similar to (9),

$$
f(t)=\sum_{k} \mathbf{c}_{l}^{*}(k) \Phi_{l, k}(t)+\sum_{j=l}^{n-1} \sum_{k} \mathbf{d}_{j}^{*}(k) \Psi_{j, k}(t),
$$

where $l=n$-(levels for decomposition) and $\mathbf{c}_{l}^{*}(k), \mathbf{d}_{j}^{*}(k)$ are row vectors.
Multiwavelets are a new addition to the body of wavelet theory. Realizable as matrix-valued filter banks leading to wavelet bases, multiwavelets offer simultaneous orthogonality, symmetry, and short support, which is not possible with scalar two-channel wavelet systems. The DGHM multiwavelets which are developed by Donovan et al. [15] are a very popular multiwavelet. They are shown in Figure 1.

Remark 1. It is important to note that the orthogonality conditions for the DGHM scaling function and the associated multiwavelet remain valid even when restricted to a compact interval. This is not true in general. It is actually another favorable property of this multiwavelet system.

The DGHM scaling functions satisfy (1) with four coefficients:

$$
\begin{array}{ll}
H_{0}=\left(\begin{array}{cc}
\frac{3}{10} & \frac{2 \sqrt{2}}{5} \\
-\frac{\sqrt{2}}{40} & -\frac{3}{20}
\end{array}\right), & H_{1}=\left(\begin{array}{cc}
\frac{3}{10} & 0 \\
\frac{9 \sqrt{2}}{40} & \frac{1}{2}
\end{array}\right), \\
H_{2}=\left(\begin{array}{cc}
0 & 0 \\
\frac{9 \sqrt{2}}{40} & -\frac{3}{20}
\end{array}\right), & H_{3}=\left(\begin{array}{cc}
0 & 0 \\
-\frac{\sqrt{2}}{40} & 0
\end{array}\right) .
\end{array}
$$

And the multiwavelets satisfy (1) with four coefficients as

$$
\begin{array}{cc}
G_{0}=\left(\begin{array}{cc}
-\frac{\sqrt{2}}{40} & -\frac{3}{40} \\
-\frac{1}{20} & -\frac{3 \sqrt{2}}{20}
\end{array}\right), \quad G_{1}=\left(\begin{array}{cc}
\frac{9 \sqrt{2}}{40} & -\frac{1}{2} \\
\frac{9}{20} & 0
\end{array}\right), \\
G_{2}=\left(\begin{array}{cc}
\frac{9 \sqrt{2}}{40} & -\frac{3}{20} \\
-\frac{9}{20} & \frac{3 \sqrt{2}}{20}
\end{array}\right), \quad G_{3}=\left(\begin{array}{cc}
-\frac{\sqrt{2}}{40} & 0 \\
\frac{1}{20} & 0
\end{array}\right) .
\end{array}
$$

GHM multiple scaling functions and multiwavelets are very popular with many advantages over scalars. They are either symmetric or antisymmetric, orthogonal, supported on $[0,1]$ and $[0,2]$, respectively, with approximation order 2 . However, it is impossible for scalar wavelets [16].

Let $\mathbf{c}_{j}(k)=\left(c_{1, j, k}, c_{2, j, k}\right)$ and $\mathbf{d}_{j}(k)=\left(d_{1, j, k}, d_{2, j, k}\right)$. By dilations (1) and (3), we have the following recursive relationship between the coefficients $\left(c_{1, j, k}, c_{2, j, k}\right)^{T}$ and $\left(d_{1, j, k}, d_{2, j, k}\right)^{T}$ :

$$
\begin{aligned}
& \left(\begin{array}{l}
c_{1, j-1, k} \\
c_{2, j-1, k}
\end{array}\right)=\sum_{n} H_{n-2 k}\left(\begin{array}{l}
c_{1, j, n} \\
c_{2, j, n}
\end{array}\right), \quad j, k \in Z \\
& \left(\begin{array}{l}
d_{1, j-1, k} \\
d_{2, j-1, k}
\end{array}\right)=\sum_{n} G_{n-2 k}\left(\begin{array}{l}
c_{1, j, n} \\
c_{2, j, n}
\end{array}\right), \quad j, k \in Z .
\end{aligned}
$$



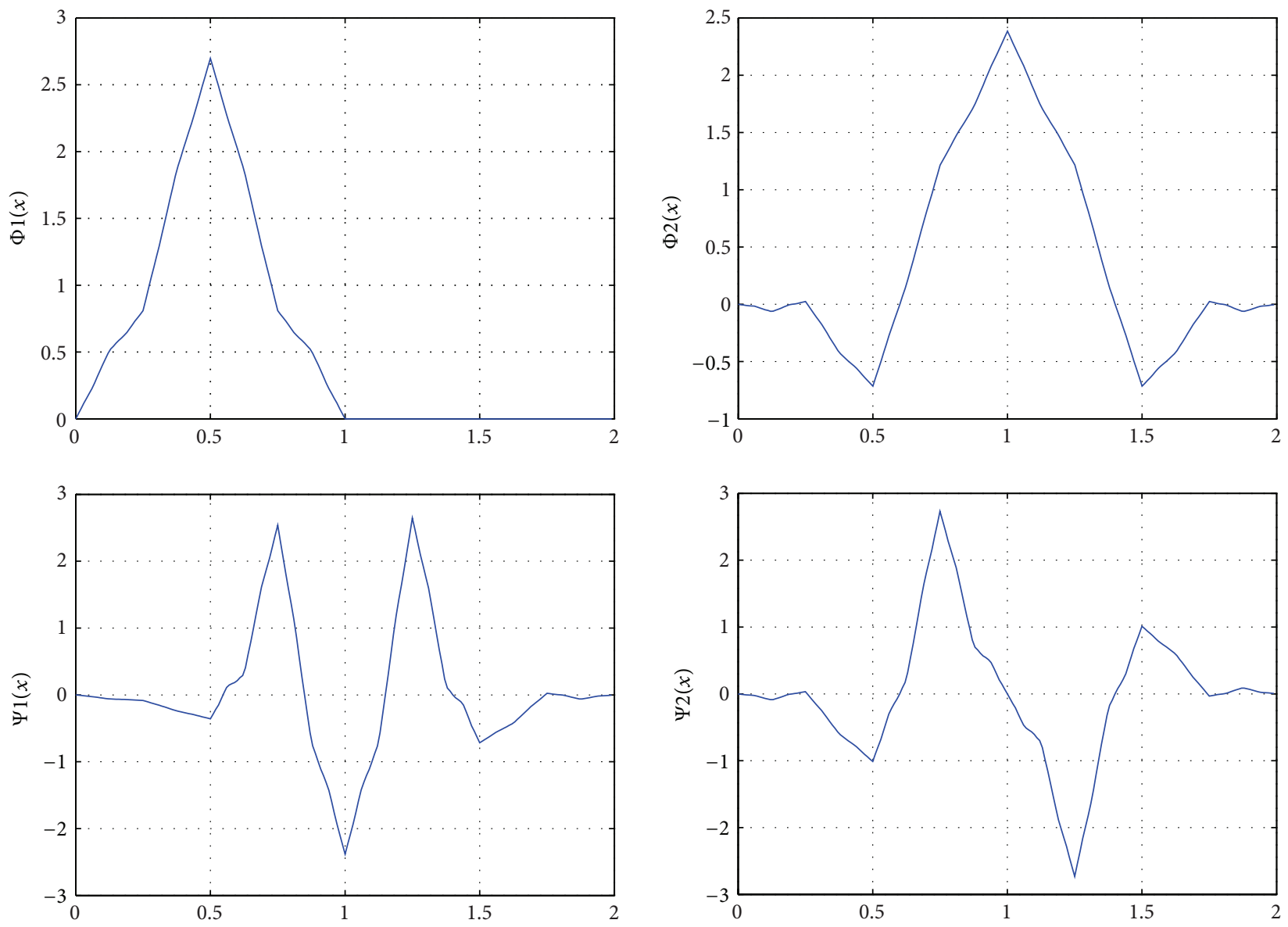

FIGURE 1: GHM multiple scaling functions (top) and multiwavelets (bottom).

Moreover,

$$
\begin{aligned}
& \left(\begin{array}{l}
c_{1, j, k} \\
c_{2, j, k}
\end{array}\right) \\
& \quad=\sum_{n}\left(H_{n-2 k}^{T}\left(\begin{array}{l}
c_{1, j-1, n} \\
c_{2, j-1, n}
\end{array}\right)+G_{n-2 k}^{T}\left(\begin{array}{l}
d_{1, j-1, n} \\
d_{2, j-1, n}
\end{array}\right)\right), \quad j, k \in Z .
\end{aligned}
$$

Due to their matrix-valued filter banks, multiwavelets differ from scalar wavelets in requiring two or more input streams. That means the multiwavelet decomposition and reconstruction are a multi-input and multioutput (MIMO) system. It is necessary to preprocess the input signal before decomposition and postprocess the output signal after reconstruction [17]. Postfilter is just the inverse matrix of prefilter.

Thus, the above decomposition and reconstruction can be represented in Figure 2. $Q(\omega)$ is the prefilter and $P(\omega)$ is the postfilter.

\section{Active Power Representation Using Multiwavelet Transform}

It is known that the active power is calculated by averaging the voltage and current product with a running window over one cycle of the fundamental frequency

$$
p=\frac{1}{\mathrm{~T}} \int_{0}^{\mathrm{T}} u(t) i(t) d t
$$

where $u(t)$ and $i(t)$ are instantaneous voltage and current, and $\mathrm{T}$ is the period of the specified fundamental frequency.

Let $t=\mathrm{T} x, u^{\prime}(x)=u(\mathrm{~T} x)$, and $i^{\prime}(x)=i(\mathrm{~T} x)$. Then (16) becomes

$$
p=\int_{0}^{1} u^{\prime}(x) i^{\prime}(x) d x .
$$

Suppose that the instantaneous voltage $u^{\prime}(x)$ and current $i^{\prime}(x)$ have been discretized and expressed using scaling functions and multiwavelets over a running unit interval $0 \leq$ $x \leq 1$. Consider

$$
\begin{gathered}
u^{\prime}(x)=\sum_{j} \mathbf{c}_{J_{0}, j}^{*} \Phi_{J_{0}, j}(x)+\sum_{j} \mathbf{d}_{J_{0}, j}^{*} \Psi_{J_{0}, j}(x), \\
i^{\prime}(x)=\sum_{j} \mathbf{a}_{K_{0}, j}^{*} \Phi_{K_{0}, j}(x)+\sum_{j} \mathbf{b}_{K_{0}, j}^{*} \Psi_{K_{0}, j}(x),
\end{gathered}
$$

where $J_{0}$ and $K_{0}$ are the coarsest resolution level of the decomposition; $\mathbf{c}_{J_{0}, j}^{*}$ and $\mathbf{a}_{J_{0}, j}^{*}$ are the approximation coefficients that represent the smoothed part of the signal; $\mathbf{d}_{J_{0}, j}^{*}$ and 


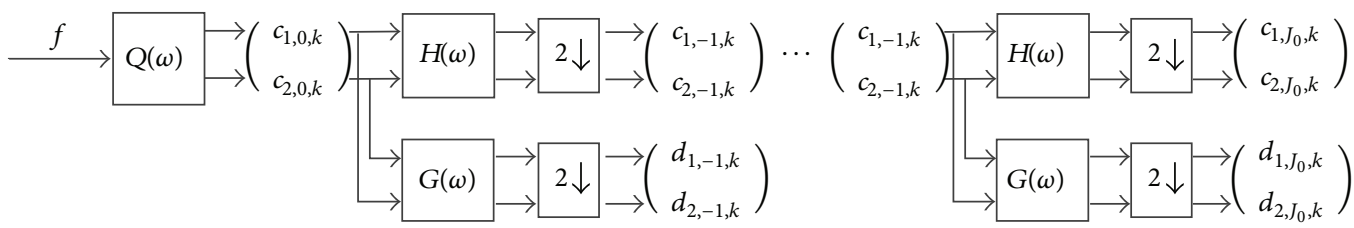

(a)

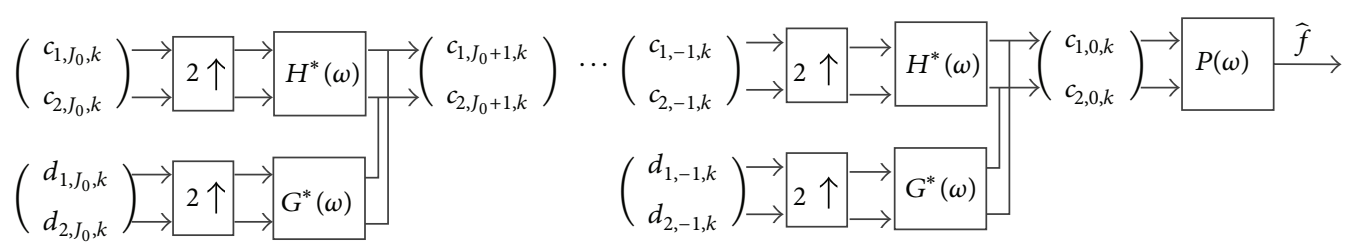

(b)

FIgURE 2: Multiwavelets (a) decomposition and (b) reconstruction $(r=2)$.

$\mathbf{b}_{J_{0}, j}^{*}$ are the detail coefficients that represent the oscillatory part of the same signal.

Substituting (18) into (17), we have

$$
\begin{aligned}
p=\int_{0}^{1} & {\left[\sum_{j} \mathbf{c}_{J_{0}, j}^{*} \Phi_{J_{0}, j}(x)+\sum_{j} \mathbf{d}_{J_{0}, j}^{*} \Psi_{J_{0}, j}(x)\right] } \\
\times & {\left[\sum_{j} \mathbf{a}_{K_{0}, j}^{*} \Phi_{K_{0}, j}(x)+\sum_{j} \mathbf{b}_{K_{0}, j}^{*} \Psi_{K_{0}, j}(x)\right] d x . }
\end{aligned}
$$

Define $\mathbf{c}_{J_{0}, j}^{*}=\left(c_{J_{0}, j, 1}, c_{J_{0}, j, 2}\right)^{T}, \mathbf{d}_{J_{0}, j}^{*}=\left(d_{J_{0}, j, 1}, d_{J_{0}, j, 2}\right)^{T}, \mathbf{a}_{K_{0}, j}^{*}=$ $\left(a_{K_{0}, j, 1}, a_{K_{0}, j, 2}\right)^{T}$, and $\mathbf{b}_{K_{0}, j}^{*}=\left(b_{K_{0}, j, 1}, b_{K_{0}, j, 2}\right)^{T}$; then (19) can be rewritten as

$$
\begin{aligned}
& p=\int_{0}^{1} \sum_{j, k}\left(c_{J_{0}, j, 1} a_{K_{0}, k, 1} \phi_{J_{0}, j, 1}(x) \phi_{K_{0}, k, 1}(x)\right. \\
& +c_{J_{0}, j, 1} a_{K_{0}, k, 2} \phi_{J_{0}, j, 1}(x) \phi_{K_{0}, k, 2}(x) \\
& +c_{J_{0}, j, 2} a_{K_{0}, k, 1} \phi_{J_{0}, j, 2}(x) \phi_{K_{0}, k, 1}(x) \\
& \left.+c_{J_{0}, j, 2} a_{K_{0}, k, 2} \phi_{J_{0}, j, 2}(x) \phi_{K_{0}, k, 2}(x)\right) d x \\
& +\int_{0}^{1} \sum_{j, k}\left(c_{J_{0}, j, 1} b_{K_{0}, k, 1} \phi_{J_{0}, j, 1}(x) \psi_{K_{0}, k, 1}(x)\right. \\
& +c_{J_{0}, j, 1} b_{K_{0}, k, 2} \phi_{J_{0}, j, 1}(x) \psi_{K_{0}, k, 2}(x) \\
& +c_{J_{0}, j, 2} b_{K_{0}, k, 1} \phi_{J_{0}, j, 2}(x) \psi_{K_{0}, k, 1}(x) \\
& \left.+c_{J_{0}, j, 2} b_{K_{0}, k, 2} \phi_{J_{0}, j, 2}(x) \psi_{K_{0}, k, 2}(x)\right) d x \\
& +\int_{0}^{1} \sum_{j, k}\left(d_{J_{0}, j, 1} a_{K_{0}, k, 1} \psi_{J_{0}, j, 1}(x) \phi_{K_{0}, k, 1}(x)\right. \\
& +d_{J_{0}, j, 1} a_{K_{0}, k, 2} \psi_{J_{0}, j, 1}(x) \phi_{K_{0}, k, 2}(x)
\end{aligned}
$$

$$
\begin{gathered}
+d_{J_{0}, j, 2} a_{K_{0}, k, 1} \psi_{J_{0}, j, 2}(x) \phi_{K_{0}, k, 1}(x) \\
\left.+d_{J_{0}, j, 2} a_{K_{0}, k, 2} \psi_{J_{0}, j, 2}(x) \phi_{K_{0}, k, 2}(x)\right) d x \\
+\int_{0}^{1} \sum_{j, k}\left(d_{J_{0}, j, 1} b_{K_{0}, k, 1} \psi_{J_{0}, j, 1}(x) \psi_{K_{0}, k, 1}(x)\right. \\
+d_{J_{0}, j, 1} b_{K_{0}, k, 2} \psi_{J_{0}, j, 1}(x) \psi_{K_{0}, k, 2}(x) \\
+d_{J_{0}, j, 2} b_{K_{0}, k, 1} \psi_{J_{0}, j, 2}(x) \psi_{K_{0}, k, 1}(x) \\
\left.+d_{J_{0}, j, 2} b_{K_{0}, k, 2} \psi_{J_{0}, j, 2}(x) \psi_{K_{0}, k, 2}(x)\right) d x .
\end{gathered}
$$

We further assume the following.

(1) The signal data is zero outside the decomposition window so that the integration range can be extended to the whole real space $\mathbb{R}$.

(2) The voltage and current signals are already in the same approximation space.

(3) The voltage and current waveforms have been decomposed to the same coarsest level; that is, $J_{0}=K_{0}$ in (20).

(4) The multiwavelet analysis of voltage and current signals employs the same type of scaling functions and orthogonal multiwavelets.

Remark 2. Assumption (1) can be satisfied very easy. For example, the periodic signals such as $\sin (\omega t)$ can satisfy assumption (1).

Assumptions (2)-(4) are decided by us. Let the voltage signal and the current signal be $\sin (\omega t)$ and $\sin (\omega t+$ $\varphi)$, respectively. They are in the space $V_{0}$ and have been decomposed to the same coarsest level $V_{-5}$ using the same multiwavelet GHM. 
Expand (20) and take into account the following orthogonal properties:

$$
\begin{aligned}
& \left\langle\Phi_{j, k}, \Phi_{m, n}\right\rangle=\left\{\begin{array}{lll}
I, & j=m, & k=n \\
\mathbf{0}, & \text { others }
\end{array}\right. \\
& \left\langle\Psi_{j, k}, \Psi_{m, n}\right\rangle=\left\{\begin{array}{ll}
I, & j=m, \\
\mathbf{0}, & \text { others }
\end{array} \quad\right. \\
& \left\langle\Phi_{j, k}, \Psi_{m, n}\right\rangle=\mathbf{0} .
\end{aligned}
$$

We get

$$
\begin{aligned}
p \mid & V_{J_{0}} \\
& =\sum_{j}\left(c_{J_{0}, j, 1} a_{J_{0}, j, 1}+c_{J_{0}, j, 2} a_{J_{0}, j, 2}+d_{J_{0}, j, 1} b_{J_{0}, j, 1}+d_{J_{0}, j, 2} b_{J_{0}, j, 2}\right) \\
& =\sum_{j} \mathbf{c}_{J_{0}, j} \mathbf{a}_{J_{0}, j}^{*}+\sum_{j} \mathbf{d}_{J_{0}, j} \mathbf{b}_{J_{0}, j}^{*} .
\end{aligned}
$$

Remark 3. The orthogonal properties (21) can effectively reduce or eliminate the aliasing phenomenon. They ensure the orthogonality of the frequency band.

Using (14), we have

$$
\begin{aligned}
p \mid V_{J} & =\sum_{j} \mathbf{c}_{J_{0}, j} \mathbf{a}_{J_{0}, j}^{*}+\sum_{k=J_{0}}^{J} \sum_{j} \mathbf{d}_{k, j} \mathbf{b}_{k, j}^{*} \\
& =p_{\Phi}+p_{\Omega_{0}}+p_{\Omega_{1}}+\cdots+p_{\Omega_{J-1}},
\end{aligned}
$$

where $p_{\Phi}=\sum_{j} \mathbf{c}_{J_{0}, j} \mathbf{a}_{J_{0}, j}^{*}$ and $p_{\Omega_{k}}=\sum_{j} \mathbf{d}_{J_{0}+k, j} \mathbf{b}_{J_{0}+k, j}^{*}$ with $k=$ $0,1, \ldots, J-J_{0}$.

From (23), we can see that, in the multiwavelet domain, the power delivered is the addition of the power calculated at each decomposition level, and the power at a decomposition level is the addition of the products of the corresponding multiwavelet coefficients.

It is interesting to note that (23) resembles to a certain extent the formula for calculating active power by wavelet [7] and by Fourier series source [18].

\section{Numerical Example}

A numerical example is considered in this section. All computations in this section are carried out by Matlab 7.8.0.347.

The example considers the case of sinusoidal voltage $\left(f_{1}=50 \mathrm{~Hz}\right)$ and a nonsinusoidal current containing the fundamental, third, and fifth harmonic components

$$
\begin{gathered}
u(t)=100 \sin (314 t), \\
i(t)=10 \sin (314 t)+2 \sin (942 t)+0.3 \sin (1570 t) .
\end{gathered}
$$

In the field of power systems, it is generally considered up to the 50th harmonic signals; that is, $f_{\text {hmax }}=f_{1} \times 50=$ $2500 \mathrm{~Hz}$. According to the Nyquist sampling theorem, the sampling frequency is greater than or equal to 2 times the highest frequency; that is, $f_{s} \geq 2 \times f_{\text {hmax }}=5000 \mathrm{~Hz}$. So $f_{s}=6400 \mathrm{~Hz}$ is selected as the sampling frequency. You can also choose the other frequencies $f_{s}$ to be greater than $5000 \mathrm{~Hz}$.

After sampling the voltage and current waveforms with a sampling frequency $\left(f_{s}=6400 \mathrm{~Hz}\right)$, a prefilter is used to produce the initial coefficients. Then the multiwavelet transform is applied in order to get the approximations and the details.

Since we want to extract the fundamental signal in original signal, it needs the fundamental signal to be in the center of the frequency bank. The lowest decomposition approximation frequency range is $0-100 \mathrm{~Hz}$, which means that the width of approximation frequency bank is $100 \mathrm{~Hz}$. The width of approximation frequency bank is $B_{a}=$ $f_{s} / 2^{l+1}$, where $l$ is the decomposition level. So we have $l=$ $\log _{2}\left(f_{s} / B_{a}\right)-1=5$. If it is further decomposed to level 6 , the fundamental signal is then at the boundary of frequency range $0-50 \mathrm{~Hz}$ and $50-100 \mathrm{~Hz}$. If the decomposition level is 4 , then the approximation frequency range of the signal is $0-200 \mathrm{~Hz}$, resulting in 3rd harmonic signal that also falls in this frequency range, and the fundamental signal cannot be separated. Thus five wavelet levels are chosen, and this represents the best number of levels for decomposition; therefore, each frequency component of the analyzed waveform can be extracted in a single band.

The voltage and current signals are analyzed using multiwavelet GHM, Daubechies (Db4, Db10), and Fourier Transform (FT). When using wavelet and multiwavelet, the boundary should be handled first. There are many handle methods such as zero padding, symmetric extension, and periodic extension. Zero padding does not preserve orthogonality. It is shown in [19] that the finite multiwavelet transform based on symmetric extension will then preserve the symmetry across scales as in the scalar case. The periodic extension approach will work for multiwavelets and will preserve orthogonality unless the data are truly periodic. Because our data are periodic, here we are using the periodic extension. When using multiwavelet, it also needs a prefilter to transfer the scalar signal into a vector. We are using the two popular prefilters, one is the oversampling [20] and other is GHM.init [17].

The calculation results of the active power using GHM with oversampling prefilter, GHM with GHM.init prefilter, Db4, Db10, and FT are listed in Table 1. We can see that an active power of about $500 \mathrm{~W}$ is obtained, and the difference is too small. Furthermore, we can see that the active power measured using multiwavelet no matter which prefilter is used is better than using wavelet (Db4 and Db10) and Fourier Transforms.

Remark 4. From the results, we can see that the active power measured using multiwavelet no matter which prefilter is used is better than using wavelet (Db4 and Db10) and Fourier Transforms. However, multiwavelet transform needs prefiltering and postfiltering for one-dimensional signals. Calculation of multiwavelet transform is greater than wavelet transform. Fast algorithm for multiwavelet transform will be our next work. 
TABLE 1: Active power calculation results.

\begin{tabular}{lcccc}
\hline Method name & Prefilter & Theory values & Calculated values & Error \\
\hline GHM & Oversampling & 500 & 500.3804 & $7.6 \times 10^{-4}$ \\
GHM & GHM.init & 500 & 500.3755 & $7.5 \times 10^{-4}$ \\
db4 & $\backslash$ & 500 & 501.8237 & $3.6 \times 10^{-3}$ \\
db10 & $\backslash$ & 500 & 499.4937 & $1.0 \times 10^{-3}$ \\
FT & $\backslash$ & 500 & 501.2168 & $2.4 \times 10^{-3}$ \\
\hline
\end{tabular}

\section{Conclusions}

A new method of active power measurement was mathematically examined in the paper. It is shown that the active power can be calculated by simply adding the products of multiwavelet coefficients without having to spend time on synthesizing the processed coefficients back to time-domain signals first and then using the traditional integration. The new method is convenient and beneficial when multiwavelet transform has to be performed in the true real-time environment or to compress electric data of large volume. The new method is better than wavelet and Fourier Transforms.

\section{Conflict of Interests}

The authors declare that there is no conflict of interests concerning the publication of this paper.

\section{Acknowledgment}

This work was partially supported by National Natural Science Foundation of China (51277043).

\section{References}

[1] W.-K. Yoon and M. J. Devaney, "Power measurement using the wavelet transform," IEEE Transactions on Instrumentation and Measurement, vol. 47, no. 5, pp. 1205-1210, 1998.

[2] A. E. Emanuel, "Powers in non-sinusoidal situations, A review of definitions and physical meaning," IEEE Transactions on Power Delivery, vol. 5, no. 3, pp. 1377-1383, 1990.

[3] X. Zhang, Y. Yan, W. Cao, and H. Wu, "A novel rational measurement method for electric energy of distorted signals," in Proceedings of the International Conference on New Trends in Information and Service Science (NISS '09), pp. 1046-1051, Beijing, China, July 2009.

[4] A. Ortiz, M. Mañana, C. J. Renedo, S. Pérez, and F. Delgado, "A new approach to frequency domain power measurement based on distortion responsibility," Electric Power Systems Research, vol. 81, no. 1, pp. 202-208, 2011.

[5] F. Vatansever and A. Ozdemir, "Power parameters calculations based on wavelet packet transform," International Journal of Electrical Power and Energy Systems, vol. 31, no. 10, pp. 596-603, 2009.

[6] X.-B. Zhang, Y.-H. Liang, and J.-R. Cui, "A novel active power measuring method for power systems with distorted signals," in Proceedings of the IEEE International Conference on Information and Automation (ICIA '08), pp. 5-9, Zhangjiajie, China, June 2008.
[7] T. X. Zhu, "A new approach to active power calculation using wavelet coefficients," IEEE Transactions on Power Systems, vol. 21, no. 1, pp. 435-437, 2006.

[8] W. G. Morsi and M. E. El-Hawary, "Reformulating power components definitions contained in the IEEE standard 14592000 using discrete wavelet transform," IEEE Transactions on Power Delivery, vol. 22, no. 3, pp. 1910-1916, 2007.

[9] S. Kaewarsa, K. Attakitmongcol, and T. Kulworawanichpong, "Recognition of power quality events by using multiwaveletbased neural networks," International Journal of Electrical Power and Energy Systems, vol. 30, no. 4, pp. 254-260, 2008.

[10] Q. Yinglin, T. Lijun, and W. Chunbao, "Power quality disturbances detection and location based on multi-wavelet and neighboring coefficient de-noising," in Proceedings of the 4th International Conference on Electric Utility Deregulation and Restructuring and Power Technologies (DRPT '11), pp. 424-427, WeiHai, China, July 2011.

[11] R. Kapoor and M. K. Saini, "Multiwavelet transform based classification of PQ events," European Transactions on Electrical Power, vol. 22, no. 4, pp. 518-532, 2012.

[12] J. S. Geronimo, D. P. Hardin, and P. R. Massopust, "Fractal functions and wavelet expansions based on several scaling functions," Journal of Approximation Theory, vol. 78, no. 3, pp. 373-401, 1994.

[13] S. G. Mallat, "Theory for multiresolution signal decomposition: the wavelet representation," IEEE Transactions on Pattern Analysis and Machine Intelligence, vol. 11, no. 7, pp. 674-693, 1989.

[14] F. Keinert, Wavelets and Multiwavelets, Chapman \& Hall/CRC, Boca Raton, Fla, USA, 2004.

[15] G. C. Donovan, J. S. Geronimo, D. P. Hardin, and P. R. Massopust, "Construction of orthogonal wavelets using fractal interpolation functions," SIAM Journal on Mathematical Analysis, vol. 27, no. 4, pp. 1158-1192, 1996.

[16] I. Daubechies, Ten Lectures on Wavelets, SIAM, Philadelphia, Pa, USA, 1992.

[17] X.-G. Xia, J. S. Geronimo, D. P. Hardin, and B. W. Suter, "Why and how prefiltering for discrete multiwavelet transforms," in Proceedings of the IEEE International Conference on Acoustics, Speech, and Signal Processing (ICASSP '96), vol. 3, pp. 1578-1581, May 1996.

[18] D. E. Scott, An Introduction to Circuit Analysis: A System Approach, McGraw-Hill, New York, NY, USA, 1987.

[19] T. Xia and Q. Jiang, "Optimal multifilter banks: design, related symmetric extension transform, and application to image compression," IEEE Transactions on Signal Processing, vol. 47, no. 7, pp. 1878-1889, 1999.

[20] A. Vasily Strela and A. T. Walden, "Signal and image denoising via wavelet thresholding: orthogonal and biorthogonal, scalar and multiple wavelet transforms," Tech. Rep. TR-98-01, Imperial College, Statistics Section, 1998. 


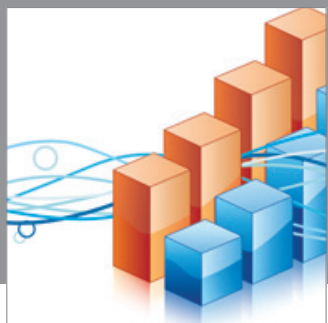

Advances in

Operations Research

mansans

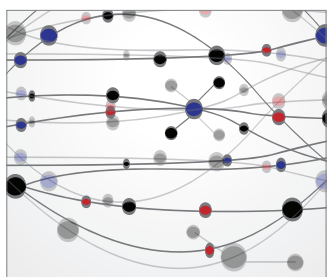

The Scientific World Journal
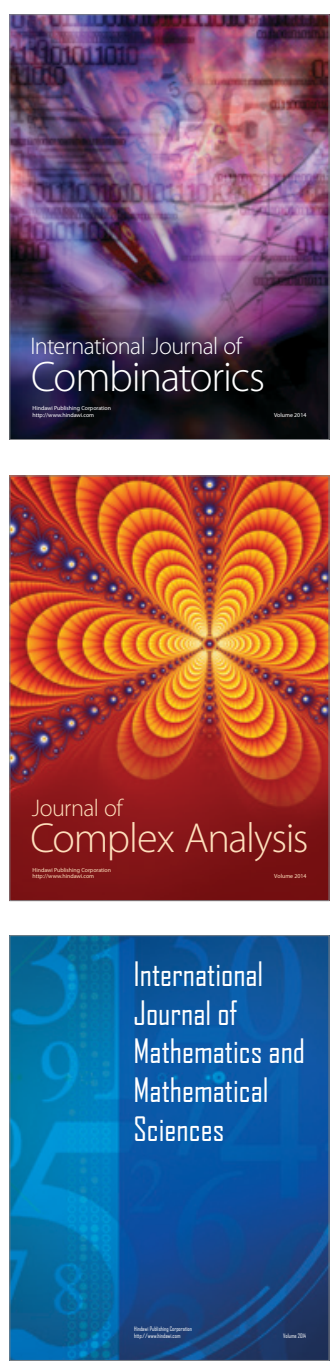
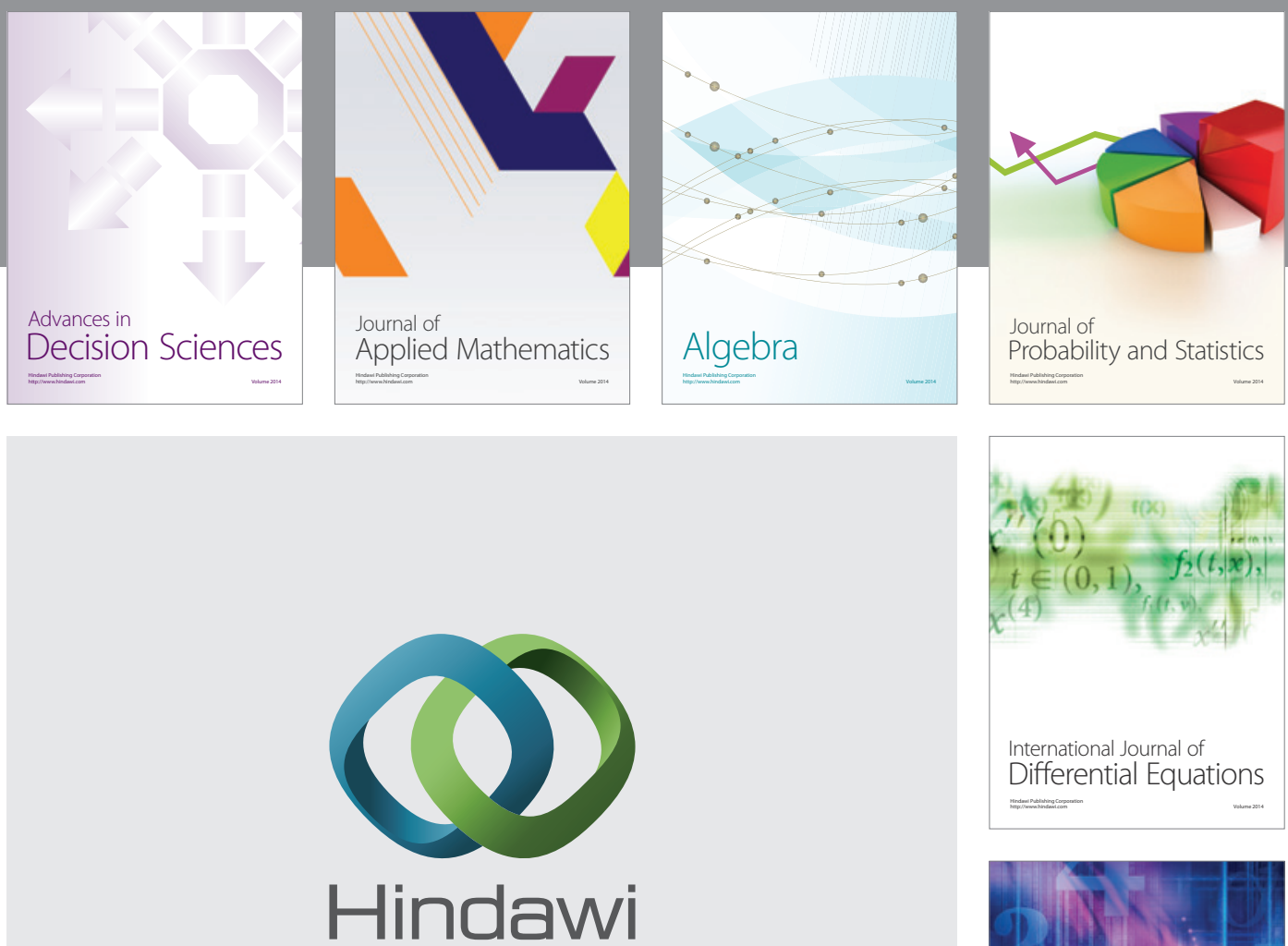

Submit your manuscripts at http://www.hindawi.com
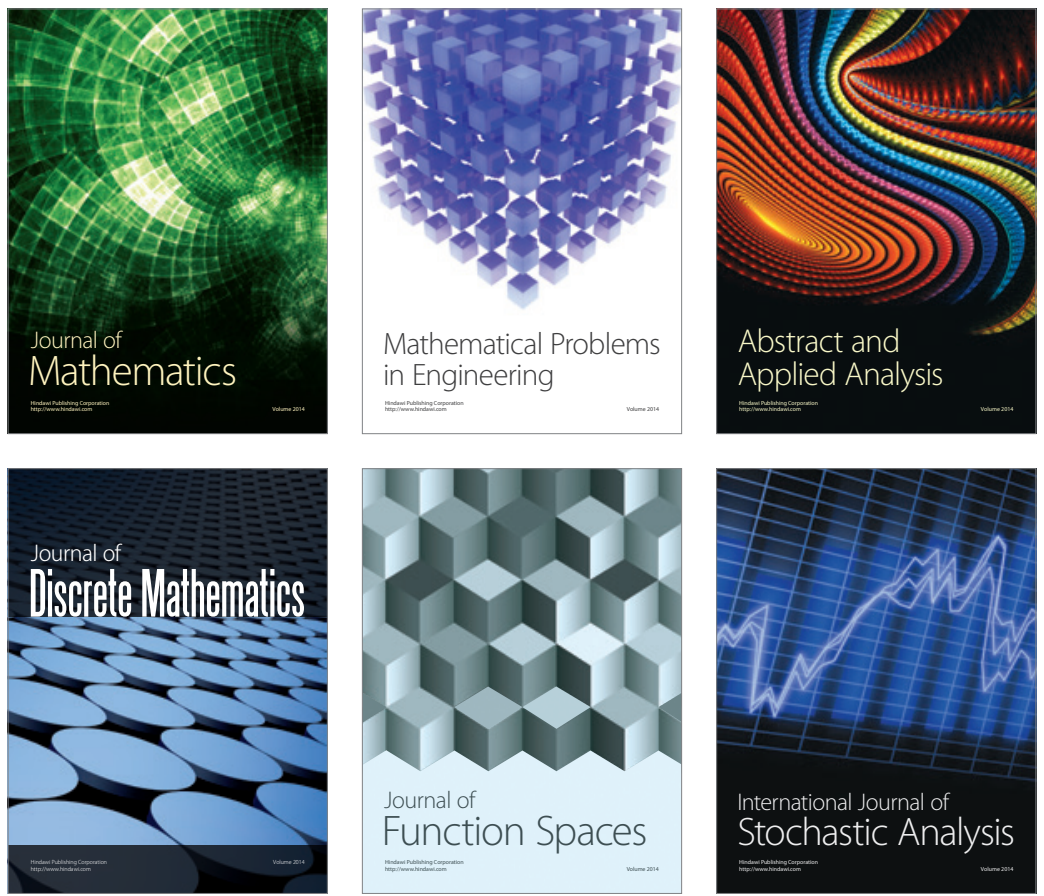

Journal of

Function Spaces

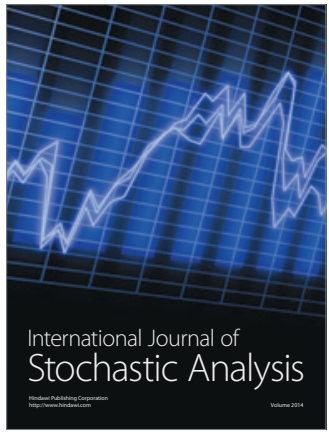

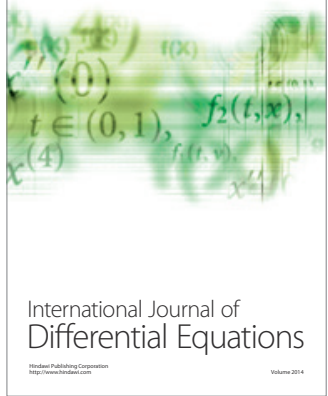
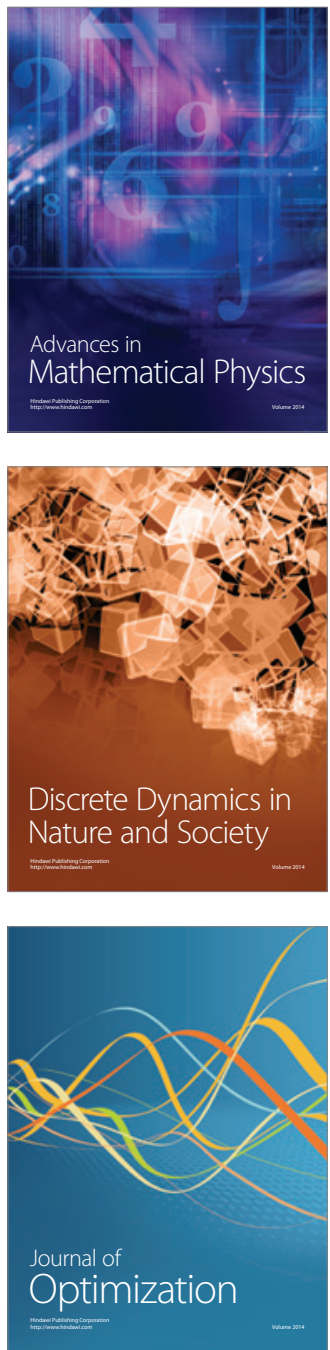